\title{
Clarification et stabilisation des vins par ultrafiltration tangentielle sur membranes minérales
}

\author{
par \\ D. POIRIER ${ }^{* * *}$, M. BENNASAR ${ }^{* *}$, B. TARODO DE LA FUENTE**, \\ J. GILLOT ${ }^{* * *}$ et D. GARCERA****
}

L'article correspondant paraîtra dans un prochain numéro de la revue I.A.A. (mai 1984).

\section{Rés u m é}

L'application de l'ultrafiltration sur membrane minérale à la clarification et la stérilisation des vins est envisagée. L'étude des caractéristiques membranaires, des paramètres hydrodynamiques et de la durée d'ultrafiltration permet de dégager des conditions optimales de travail.

L'étude du diamètre moyen des pores de la membrane montre que le diamètre de $0,2 \mu \mathrm{m}$ est suffisant pour que le filtrat recueilli soit biologiquement stable et conforme aux normes en vigueur (moins de 10 germes par litre), et que le débit d'ultrafiltrat est peu affecté par la taille des pores.

Une vitesse tangentielle élevée améliore nettement les débits de perméation sans nuire à la qualité du produit final.

De même, au moins pour les faibles valeurs, la pression facilite les transferts de matières à travers le filtre sans entraîner de relargage de solutés au cours du temps.

La température favorise également la filtration, mais provoque une détérioration des qualités organoleptiques du vin.

\footnotetext{
* Imeca-Oenologie, Z.I. - 34800 Clermont-L'Hérault.

** Groupe de recherche et de développement sur les membranes, Centre de génie et technologie alimentaire, Laboratoire de technologie alimentaire, U.S.T.L., place Eugène-Bataillon - 34060 Montpellier.

*** Ceraver, Usine de Bazet, B.P. 113 - 65001 Tarbes.
} 
Dans les conditions opératoires optimales déterminées lors de cette étude : diamètre des pores $0,2 \mu \mathrm{m}$, vitesse et pression maximales compatibles avec les réalités industrielles, température inférieure à $30^{\circ} \mathrm{C}$, le débit d'ultrafiltration à l'équilibre est de l'ordre de $80 \mathrm{l} / \mathrm{h} . \mathrm{m}^{2}$, et il reste relativement stable pendant $8 \mathrm{~h}$; la stérilité et la clarification du perméat sont systématiques.

La technique a été appliquée avec succès à différents types de vins (rouge, rosé, blanc), prétraités ou non, et issus de divers procédés de vinification.

L'analyse des constituants chimiques du vin avant et pendant l'ultrafiltration permet de mettre en évidence le rôle majeur des polysaccharides, des polyphénols et des matières azotées dans le colmatage. L'absence de rétention de l'éthanol garantit la qualité commerciale du produit.

Enfin, un cycle de nettoyage en place, simple et économique, mettant à profit la grande résistance mécanique, chimique et thermique de ce nouveau matériau minéral, est déterminé. 